\title{
Impact of the Location of Myometric Measurement Points on Skeletal Muscle Mechanical Properties Outcomes
}

\author{
J. Mencel, J. Marusiak, A. Jaskólska, A. Jaskólski, K. Kisiel-Sajewicz \\ Department of Kinesiology, Faculty of Physiotherapy, University School of Physical Education in Wroclaw, \\ Wroclaw, Poland
}

\author{
CORRESPONDING AUTHOR: \\ Joanna Mencel \\ Department of Kinesiology \\ Faculty of Physiotherapy \\ University School of Physical Education in \\ Wroclaw \\ al. I.J. Paderewskiego 35 \\ 51-612 Wroclaw Poland \\ E-mail: joanna.mencel@awf.wroc.pl \\ DOI: \\ 10.32098/mltj.03.2021.18
}

LEVEL OF EVIDENCE: 4

\begin{abstract}
SUMMARY
Background. The aim of this study was to assess to what extent changing the measurement point affected the results of myometric parameters describing muscle mechanical properties assessed by a MyotonPRO ${ }^{\circledR}$ device in three skeletal muscles: the biceps brachii $(\mathrm{BB})$, tibialis anterior $(\mathrm{TA})$ and rectus femoris $(\mathrm{RF})$. We hypothesised that muscle mechanical properties would change differently as the myometry probe was moved towards the proximal or distal part of a muscle.

Methods. Sixteen untrained, healthy, young male students participated in the study. Myometric frequency, stiffness, decrement, relaxation and creep parameters were measured in the $\mathrm{BB}, \mathrm{RF}$ and $\mathrm{TA}$ of the right and left sides of the body at five measurement points: the central point of a muscle, and points shifted by $10 \%$ and $20 \%$ of the muscle length proximally and distally from the central point. A multivariate: 5 (measurement points) $\times 3$ (muscle) analysis of variance (MANOVA) for each of the parameters separately was used for the main analysis.

Results. MANOVA showed that measurement point (the location), muscle and interaction between measurement point and muscle have an impact on all measured parameters $(\mathrm{P}<0.05)$ except interaction between measurement point and muscle in relaxation parameter $(\mathrm{P}>0.05)$.

Differences in myometric values were shown for both measurement points located distant from each other, as well as for those located adjacent to each other.

Conclusions Our results emphasized the variation in muscle properties along the long axis of chosen skeletal muscles expressed by myometry outcomes.
\end{abstract}

KEY WORDS

Creep; elasticity; muscle-tendon unit; stiffness; tone; myoton.

\section{BACKGROUND}

\section{Measurement point for myometric assessment of muscle properties}

It was asserted that when muscle mechanical properties are tested using myometry, the "measuring point is the most prominent point of the muscle at contraction" (1). There was a suggestion that the middle part of the muscle belly should be the particular myometric measuring point (2); however, studies reported in the literature have employed different places/points of measurements for assessment of muscle properties in different muscles.
In the case of the biceps brachii (BB) muscle some authors performed myometric measurements at a point midway between the most anterior aspect of the lateral tip of the acromion and the mid-cubital fossa $(3,4)$, following a gentle, resisted isometric muscle contraction to identify the middle of the muscle belly in a technique similar to that used by others $(5,6)$. In another experiment by Agyapong-Badua et al. (7) the measurements for the $\mathrm{BB}$ were taken at three-quarters of the distance between the lateral tip of the acromion and the mid-cubital fossa. Meanwhile, lateral part of the muscle in the middle of the arm was used for measurement for the long head of the $\mathrm{BB}$ or $75 \%$ of the 
distance distally from the anterior aspect of the lateral acromion to the mid-cubital fossa was used for this purpose (8). For assessment of the mechanical properties of the rectus femoris $(\mathrm{RF})$ muscle, some authors chose a point two-thirds of the distance between the anterior superior iliac spine and the superior pole of the patella over the muscle belly $(3-5,9,10)$. Schoenrock et al. (11) measured RF stiffness at the end of the second third distance on a virtual line between the anterior superior iliac spine and the patella, with the body in the supine position, while the paper by Zinder and Padua (12) did not provide any details describing the position of $\mathrm{RF}$ measurement.

In a study by Schoenrock et al. (11), the measurement point for the tibialis anterior (TA) was positioned $1.5 \mathrm{~cm}$ laterally from the end of the proximal third of the frontal tibial line. Meanwhile Lo et al. (4) took measurements of the TA at the upper two thirds of the distance between the lateral condyle of the tibia and the medial cuneiform. Keeping in mind the suggestions of Gapeyeva and Vein (2) that the middle part of the muscle belly is an optimal location for assessment of myometric mechanical properties and taking into account the literature variation in reporting the locations of such points, it is necessary to assess whether changing the myometric measurement point along the long axis of the muscle belly in various skeletal muscles affects the measurements of mechanical properties outcomes and in what extend.

\section{Muscle mechanical properties}

Mechanical properties of skeletal muscle are measured using different equipment such as shear wave ultrasound elastography, the free oscillation technique, magnetic resonance elastography. However, lately more and more authors have measured the mechanical properties of skeletal muscle using a less expensive portable method called myometry by means of a MyotonPRO ${ }^{\circledR}$ or Myoton-3 device. Importantly it has been shown that the measurements obtained with the $\mathrm{Myoton} \mathrm{RO}{ }^{\otimes}$ have high reproducibility and reliability in study of healthy subjects and the clinical population as well (4-13).

Mechanical properties of skeletal muscle may not be the same along the long axis due to muscle architecture and anatomy and thus monitoring muscle mechanical properties at a single location may not reflect the heterogeneous properties of the muscle. At the end of a muscle there are more collagen fibres that form the aponeurosis and tendon close to the proximal or distal part of the muscle and the collagen content has been found to be closely associated with skeletal muscle passive stiffness (14). Collagen stiffness (and thus tendon stiffness) is higher than skeletal muscle stiffness (15, 16). Collagen makes up for $60-80 \%$ of tendon's tissue dry weight. It is formed as a set of parallel bundles of collagen fibrils. Collagen is also an important part of skeletal muscle extracellular matrix. It is a vital component of the connective tissue sheaths that enfold each of skeletal muscle's layer. The function of these layers is to structurally support and to assist muscles' force transfer to bone (17). The total compliance of the muscle-tendon unit could be mainly associated with the tendon because it has been found that the tendons are much longer than the muscle fascicles (18). Taking into consideration the fact that collagen fibres are not evenly distributed within a muscle and the differences in muscle architecture between different muscles, one can expect differences in the mechanical properties of skeletal muscle along their long axis that may vary among muscles. However there is only one study assessing the effect of shifting the measurement point along a muscle by $1 \mathrm{~cm}$ (vertically and horizontally) on three (of five) myometric parameters characterizing the mechanical properties of the $\mathrm{BB}$ and RF muscles (7).

Due to discrepancies in the literature related to the choice of muscle point for measuring the mechanical properties of muscles using myometry, our study aimed to investigate whether and to what extent changing the measurement point, proximally or distally from the central point, affected the results of myometric parameters in the $\mathrm{BB}, \mathrm{TA}$ and $\mathrm{RF}$ muscles. Taking into consideration the potential differences in distribution of collagen fibres within a muscle related to its anatomy, architecture and function as described above, we hypothesised that the mechanical properties of a given skeletal muscle may exhibit differences towards the proximal and distal ends of the muscle. In contrast to the study of Agyapong-Badu et al. (7) we extended the knowledge by shifting the measurement point relative to the approximate length of the muscle by 10 and $20 \%$ toward the proximal and distal ends of the muscle from its central point above the muscle belly. In our opinion, usage of an absolute value (7) in shifting the distance by $1 \mathrm{~cm}$ in muscles differing in length might give a different or fuzzy picture (although this does not detract from the value of their study). Thus, the novelty of our study is that we report all five myometric parameters of three muscles (BB - upper limb, RF and TA - lower limb) at five points distant (by $10 \%$ of muscle length) from each other. The results of our study may have practical application in the context of mechanical properties measured using myometry.

\section{MATERIALS AND METHODS}

\section{Subjects}

Sixteen untrained, young, healthy, right-hand- and leg-dominant male students (age: $21.2 \pm 0.6$ years, body mass: $77.1 \pm$ $9.8 \mathrm{~kg}$, body height: $1.80 \pm 0.07 \mathrm{~m}$ ) participated in this study. 
The exclusion criteria were injury to the upper or lower limbs, chronic disease requiring medication, and skin conditions that contraindicated taking a measurement or participation in competitive training. Upper and lower limb dominance were assessed by the Edinburgh Inventory Questionnaire (19) and the kick-ball test (20) respectively. All participants were well informed of the study's aim and procedure. Informed consent was obtained from the participants prior to the experiment. The study was approved by the ethical committee of the University School of Physical Education in Wroclaw. It was carried out in accordance with the Declaration of Helsinki and meets the ethical standards of the journal (19).

\section{Methods}

\section{Experimental procedures}

All participants were placed in the supine position, lying with their upper limb extended along the trunk and in supination and the lower limb extended and in the intermediate position. Soft towels were used to help participants to lie in this position and relax during the experiment.

\section{Identification of measurement points}

Before myometric measurements of the $\mathrm{BB}, \mathrm{TA}$ and $\mathrm{RF}$ muscles, five points on each muscle were identified. For this purpose an approximate length of the muscle along the long axis was measured as follows. For the BB muscle, it was measured from the axillary fossa to the cubital fossa. For the TA it was half the distance from the lateral knee joint space to the most anterior portion of the lateral ankle, and in the case of the RF it was from the most anterior part of the anterior superior iliac spine to the superior border of the patella. The central points of the BB (short head) and TA were located above the central part of the muscle belly, and two-thirds of the distance between the points described above for the $\mathrm{RF}$ as described in the articles cited above $(2,13)$ and in the guidelines for users from the Myoton company.

When the subject was positioned correctly, the following five points above the selected muscles bilaterally were identified (first for the right side and then for the left side): a central point and two points above the central (two proximal points) and two points below the central (two distal points) with a distance $10 \%$ of the muscles' measured approximate length (described above) between them. Thus, the distance between all the points for each muscle was $10 \%$ of their measured approximate length. They were: point 1 : $20 \%$ of a muscle length above the central point (here and after: proximal 20\%); point 2: $10 \%$ above the central point (proximal 10\%), point 3: the central point (central); point 4: $10 \%$ below the central point (distal $10 \%$ ), and point 5
- $20 \%$ of a muscle length below the central point (distal $20 \%$ ). Before the points were marked using a skin-safe pen, the investigator, always the same experienced physiotherapist, used palpation to check that the place was correctly chosen above the muscle of interest.

\section{Myometric measurements}

The next step involved myometric measurement, before which the tested subjects received oral instruction to relax. The testing end of the MyotonPRO ${ }^{\circledR}$ device was placed perpendicular to the skin surface above the marked point, and the device was lowered into the measurement position and held steadily. During this time, the device automatically performed a series of 10 mechanical taps, from which the mean value of the muscle response was used to calculate the five parameters. The myometric measurements were taken by one investigator.

\section{Myometric parameters}

Five myometric parameters were analysed. The first three are more often reported in the literature: frequency (F-MYO, $[\mathrm{Hz}]$ ), which characterizes non-neural tone; transverse stiffness $(\mathrm{S}-\mathrm{MYO},[\mathrm{N} / \mathrm{m}])$, indicating the ability of the muscle to resist an external force that modifies its shape, and decrement (D-MYO, $[\log ]$ ), which characterizes elasticity (the ability of a muscle to recover its shape after being deformedthe lower the value, the higher the elasticity and the less the damping of the tissue's oscillation). Two other parameters are rarely documented. These were relaxation time of mechanical stress (R-MYO, [ms]) that is the time needed for a reference amount of deformation to occur under a suddenly-applied reference load, and the ratio of relaxation time to deformation time, characterizing creep (C-MYO, [Deborah number]). In more fluid-like materials, less time was required for flow (shorter relaxation time), giving a lower Deborah number. Generally, the criteria for the outcome interpretation were: the higher the values of F-MYO and S-MYO, the greater the tension and stiffness of the examined soft tissue structure at dedicated body measurement points. The lower the D-MYO value the smaller the dissipation of mechanical energy during oscillation and the higher the elasticity of the muscle (1). The lower the R-MYO and C-MYO value, the higher the tension or stiffness.

\section{Statistical analysis}

Data were analysed using SPSS 22.0 software (IBM, Armonk, NY, USA) with alpha set at $\mathrm{P} \leq 0.05$. The Shapiro-Wilk test was performed to estimate the distribution of values. As many studies $(1,10,14)$ have shown no statistically significant differences in the measured parameters between body 
sides, we also checked for such potential differences between the right and left sides of the body using the $t$-test or the Wilcoxon signed-rank test (for 13 of 75 pairs) depending on the normality of the distribution. In agreement with those studies, we found in 16 subjects no significant difference between the sides of the body and thus the number of cases used in further calculations was $\mathrm{n}=32$ (16 for the right and 16 for the left side). A multivariate: 5 (measurement points) $\times 3$ (muscles) analysis of variance (MANOVA) for each of the parameters separately (F-MYO, D-MYO, S-MYO, R-MYO, C-MYO) was used for the main analysis. The Greenhouse-Geisser correction was applied where sphericity was violated and follow-up comparisons were conducted using Bonferroni-adjusted multiple comparisons. The alpha level for measurement points comparisons has been adjusted to the level of 0.01 , and for muscles comparisons to the level of 0.01666667 . The partial eta-squared $\left(\eta_{p}^{2}\right)$ test was used as a measure of effect size for MANOVA. Data are presented as means \pm standard deviations.

\section{RESULTS}

MANOVA showed that measurement point (the location), muscle and interaction between measurement point and muscle have an impact on all measured parameters $(\mathrm{P}<$ $0.05)$, except interaction between measurement point and muscle in R-MYO (table I). The $F$ values and effect size for analysed factors are reported in table I. The observed powers of tests mentioned above were bigger than 0.8 (except interaction between measurement point and muscle in C-MYO and R-MYO).

\section{Comparison between measurement points}

\section{Frequency (F-MYO)}

In the $\mathrm{BB}$ muscle, frequency increased point-by-point being the lowest at two proximal points and the highest at distal
$20 \%$, and compared to the distal $20 \%$ the values at all other points were significantly lower $(\mathrm{P}<0.01)$. The $\mathrm{BB}$ frequency values at two proximal points were also significantly lower than the frequency value at distal point $10 \%(P<0.01)$. In the TA muscle the frequency values at distal $10 \%$ and distal $20 \%$ were significantly higher than values at the proximal $10 \%$ and proximal $20 \%$ points $(\mathrm{P}<0.01)$. There was also a significant difference between central and distal $10 \%$ point $(\mathrm{P}<0.01)$. In the RF muscle there was no difference between points $(P>0.01)$ (table II).

\section{Stiffness (S-MYO)}

In the BB muscle there was no difference in stiffness between the central point and others $(\mathrm{P}>0.01)$, while the two proximal points were significantly lower than the values at the two distal points $(P<0.01)$. In the TA muscle the values of stiffness at the two proximal points and at the central point were significantly lower than the values at the two distal points $(\mathrm{P}<0.01)$. In the $\mathrm{RF}$ muscle there was no statistically significant difference between measurement points $(\mathrm{P}>$ 0.01) (table II).

\section{Decrement (D-MYO)}

In the BB muscle the value of decrement at proximal $20 \%$ was significantly lower compared central and distal $10 \%$, and the value at distal $20 \%$ was lower than values at central and distal $10 \%(\mathrm{P}<0.01)$. In the TA muscle there were no statistically significant difference between points $(\mathrm{P}>0.01)$ with the highest value at the central point (table II). In the RF muscle, decrement decreased point-by-point from the highest value at proximal $20 \%$ to the lowest at distal $20 \%$, with significantly higher values at the proximal $20 \%$ point compared to the central, distal $10 \%$ and distal $20 \%$ points, and with significantly higher values at the proximal $10 \%$ point than at distal $10 \%$ and distal $20 \%$ points $(\mathrm{P}<0.01)$. In addition, the decrement value of the RF was significantly higher in the central point than at the distal $20 \%$ point ( $\mathrm{P}$ $<0.01$ ) (table II).

Table I. The results of MANOVA with F value for measurement point (five locations: proximal $20 \%$, proximal 10\%, central, distal 10\%, distal 20\%), muscle (three muscles: biceps brachii [BB], rectus femoris [RF], tibialis anterior [TA]) and interaction between point and muscle for five myometric parameters: $\mathrm{F}-\mathrm{MYO}=$ frequency, S-MYO = stiffness, D-MYO = decrement, $\mathrm{R}-\mathrm{MYO}=$ relaxation and $\mathrm{C}-\mathrm{MYO}=$ creep. The effect size is presented as partial eta-squared $\left(\eta_{\mathrm{p}}^{2}{ }_{\mathrm{p}}\right.$.

\begin{tabular}{llll}
\hline Parameter & Measurement point & Muscle & Measurement point $\times$ Muscle \\
\hline F-MYO & $\mathrm{F}(1.97,57.22)=25.219, \mathrm{p}<0.01=0.433$ & $\mathrm{~F}(1.44,41.76)=615.89, \mathrm{p}<0.01=0.956$ & $\mathrm{~F}(2.08,60.30)=6.21, \mathrm{p}=0.03=0.168$ \\
\hline S-MYO & $\mathrm{F}(3.05,94.47)=33.45, \mathrm{p}<0.01=0.519$ & $\mathrm{~F}(1.50,46.63)=601.25, \mathrm{p}<0.01=0.951$ & $\mathrm{~F}(4.13,128.12)=12.33, \mathrm{p}<0.01=0.285$ \\
\hline $\mathrm{D}-\mathrm{MYO}$ & $\mathrm{F}(2.17,67.27)=53.44, \mathrm{p}<0.01=0.633$ & $\mathrm{~F}(2,62)=271.49, \mathrm{p}<0.01=0.898$ & $\mathrm{~F}(3.16,98.07)=29.47, \mathrm{p}<0.01=0.487$ \\
\hline R-MYO & $\mathrm{F}(2.83,87.69)=47.68, \mathrm{p}<0.01=0.678$ & $\mathrm{~F}(2,62)=563.57, \mathrm{p}<0.01=0.952$ & $\mathrm{~F}(2.81,87.02)=2.47, \mathrm{p}=0.071=0.038$ \\
\hline C-MYO & $\mathrm{F}(3.00,93.03)=70.39, \mathrm{p}<0.01=0.694$ & $\mathrm{~F}(2,62)=557.66, \mathrm{p}<0.01=0.947$ & $\mathrm{~F}(4.38,136.02)=4.12, \mathrm{p}=0.03=0.117$ \\
\hline
\end{tabular}


Table II. Mean value \pm SD of five myometric parameters (F-MYO, S-MYO, D-MYO, R-MYO and C-MYO) for three tested muscles (BB, RF and TA) with its percentage changes with respect to the central point (in brackets) and results of comparison between five measurement points (points 1 to 5) for each of the parameters within the analysed muscles.

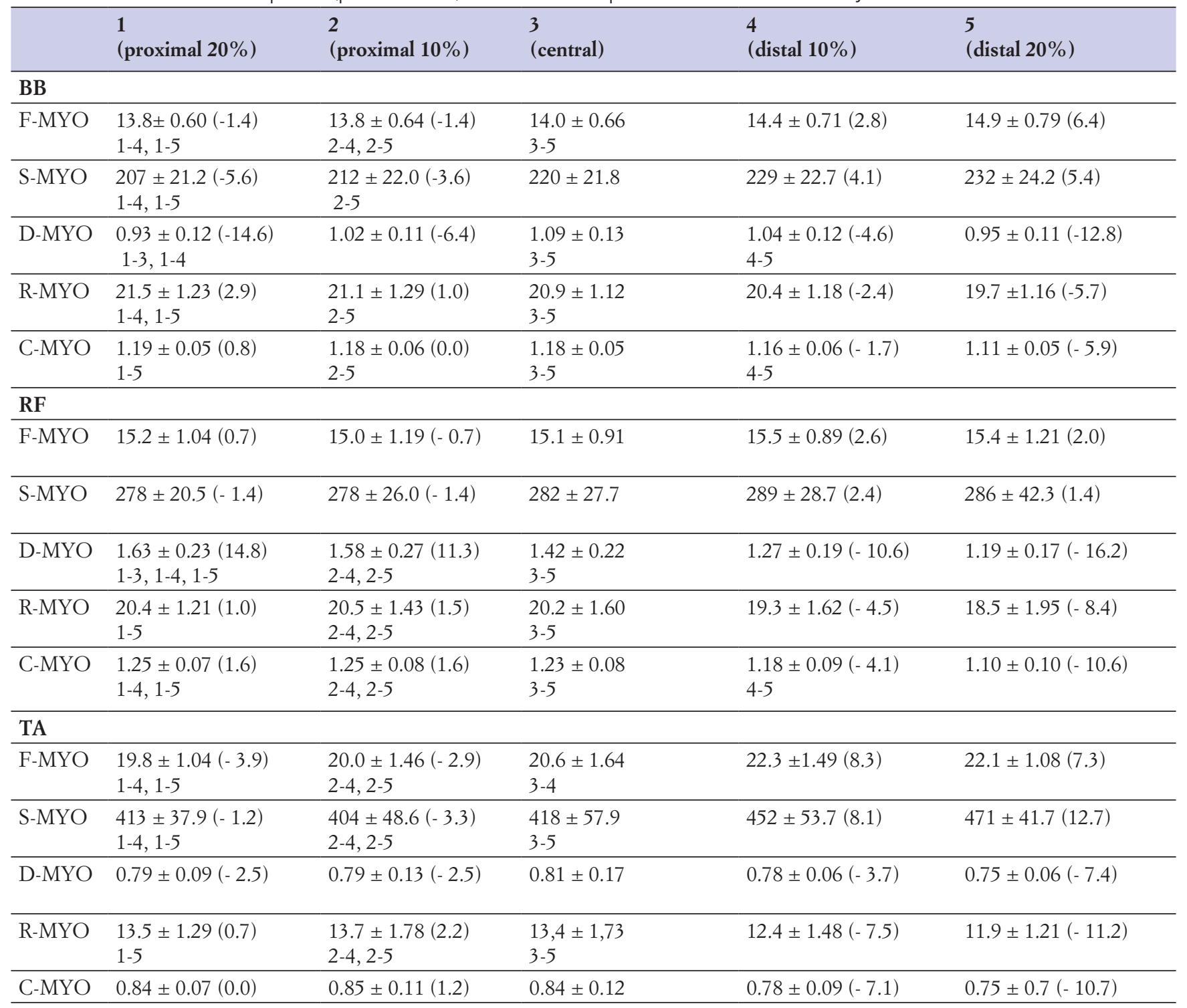

Myometric parameters: F MYO = frequency [Hz]; S-MYO: stiffness [N/m]; D-MYO: decrement [log]; R-MYO: relaxation [ms]; C-MYO: creep [Db]; BB: biceps brachii muscle; RF: rectus femoris muscle; T: tibialis anterior muscle; '- in brackets means that the value was lower compare to that for the central point. Below means \pm SD there were statistically significant differences in myometric values between points, for example 1-4 means statistically significant difference $(\mathrm{P}<0.01)$ between point 1 and point 4 .

\section{Relaxation (R-MYO)}

The lowest value of relaxation was at distal $20 \%$ points in all three muscles (BB, RF and TA muscle). In all tested muscles this distal $20 \%$ point value was significantly lower than values at proximal $20 \%$, proximal $10 \%$ and central points $(\mathrm{P}<0.01)$ while the distal $10 \%$ point value was significantly lower than the value at proximal 20\% $(\mathrm{P}<0.01)($ table II).
In addition, for the TA and RF muscles, the values at the distal $10 \%$ point were significantly lower than the values at the proximal 10\% $(\mathrm{P}<0.01)$ (table II).

\section{Creep (C-MYO)}

The smallest creep value at the distal $20 \%$ point was found in all three muscles (BB, RF, TA). In the BB and RF muscles 
the creep value at the distal $20 \%$ point was significantly lower than at other measurement points $(\mathrm{P}<0.01)$ (table II). Also, in the RF muscle the creep value at proximal $20 \%$ and proximal $10 \%$ was significantly greater than that at the distal $10 \%$ point $(\mathrm{P}<0.01)$ (table II). In the TA muscle there was no statistically significant difference between the central point and the other points $(\mathrm{P}>0.01)($ table II).

\section{Comparison between muscles}

\section{Frequency (F-MYO)}

The frequency value was significantly higher in the TA muscle than in the RF and BB muscles $(\mathrm{P}<0.02)$ (figure 1) at all measurement points. The differences between the $\mathrm{BB}$ and $\mathrm{RF}$ were not statistically significant at the distal $10 \%$ and distal $20 \%$ points $(\mathrm{P} \geq 0.02)$; however, the differences were statistically significant for the proximal $20 \%$, proximal $10 \%$ and central points $(\mathrm{P}<0.02)$ (figure 1$)$. This meant that generally the TA muscle had the highest muscle tone while the $\mathrm{BB}$ had the lowest (figure 1).

\section{Stiffness (S-MYO)}

Stiffness at each measured point was significantly higher in the TA compared to the two other muscles and the stiffness of the $\mathrm{RF}$ was higher than that of the $\mathrm{BB}$ at each measured point $(\mathrm{P}$ $<0.02$ ) (figure 2). This meant that generally the TA muscle had the highest muscle stiffness while the BB had the lowest (figure 2).

\section{Decrement (D-MYO)}

The decrement at each measurement point was the highest in the RF muscle (significantly higher value than in TA and BB, P $<0.02$ ) and the D-MYO values were significantly higher at each measurement point in the $\mathrm{BB}$ vs the $\mathrm{TA}(\mathrm{P}<0.02)$ (figure 3$)$. The results indicated that the TA was characterized by the greatest elasticity while the RF had the smallest along all measured points.

\section{Relaxation (R-MYO)}

Relaxation was significantly lower in the TA muscle than in the $\mathrm{BB}$ of the RF muscles at each measurement point $(\mathrm{P}<$ 0.02 ) (figure 3 ). The R-MYO values were significantly higher in $\mathrm{BB}$ vs RF muscle at proximal $20 \%$, distal $10 \%$ and distal $20 \%$ points (figure 4 ).

\section{Creep (C-MYO)}

Creep was the lowest in the TA muscle being significantly lower than in either $\mathrm{RF}$ or $\mathrm{BB}$ muscle at each measurement point $(\mathrm{P}<0.02)$ (figure 4$)$. The $\mathrm{C}-\mathrm{MYO}$ value was significantly higher in the $\mathrm{RF} v$ s the $\mathrm{BB}$ muscle only at the $10 \%$ proximal point $(\mathrm{P}<0.02)$ (figure 5$)$.

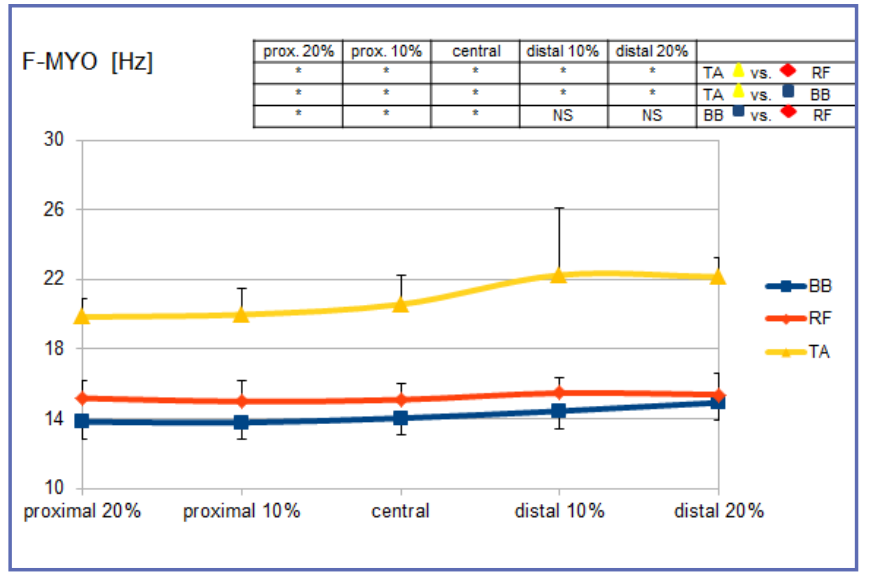

Figure 1. Mean values \pm SD of myometric frequency (F-MYO [Hz]) at five measurement points (proximal 20\%, proximal $10 \%$, central point, distal $10 \%$ and distal $20 \%$ ) for biceps brachii (BB, blue), rectus femoris (RF, red) and tibialis anterior (TA, yellow) muscles and results of pair comparison between muscles (in the upper right corner).

*Statistically significant difference $(\mathrm{P}<0.02)$; NS: not significant. Notice that $\mathrm{Y}$ axis does not start at 0 to improve resolution when illustrating differences between muscles.

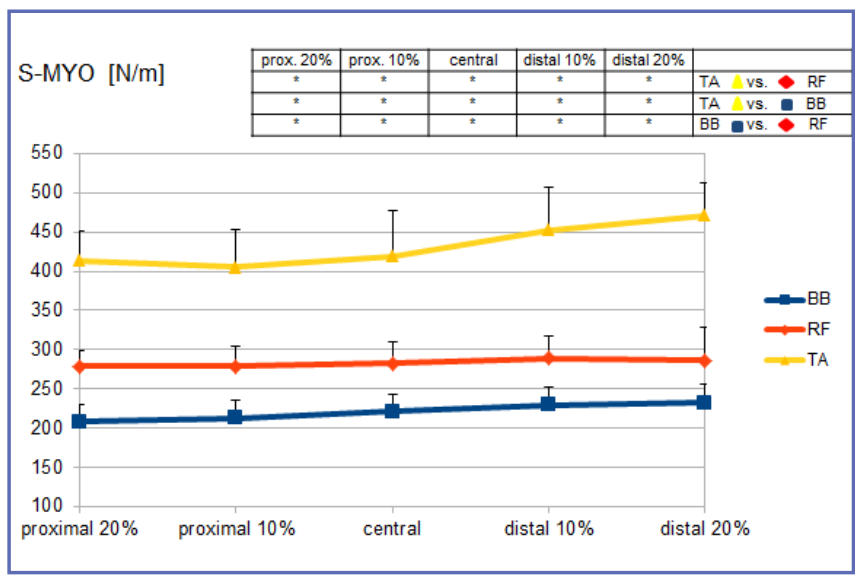

Figure 2. Mean values \pm SD of myometric stiffness (S-MYO $[\mathrm{N} / \mathrm{m}]$ ) at five measurement points (proximal 20\%, proximal $10 \%$, central point, distal $10 \%$ and distal $20 \%$ ) for biceps brachii (BB, blue), rectus femoris (RF, red) and tibialis anterior (TA, yellow) muscles and results of pair comparison between muscles (in the upper right corner).

*Statistically significant difference $(\mathrm{P}<0.02)$; NS: not significant. Notice that $\mathrm{Y}$ axis does not start at 0 to improve resolution when illustrating differences between muscles. 


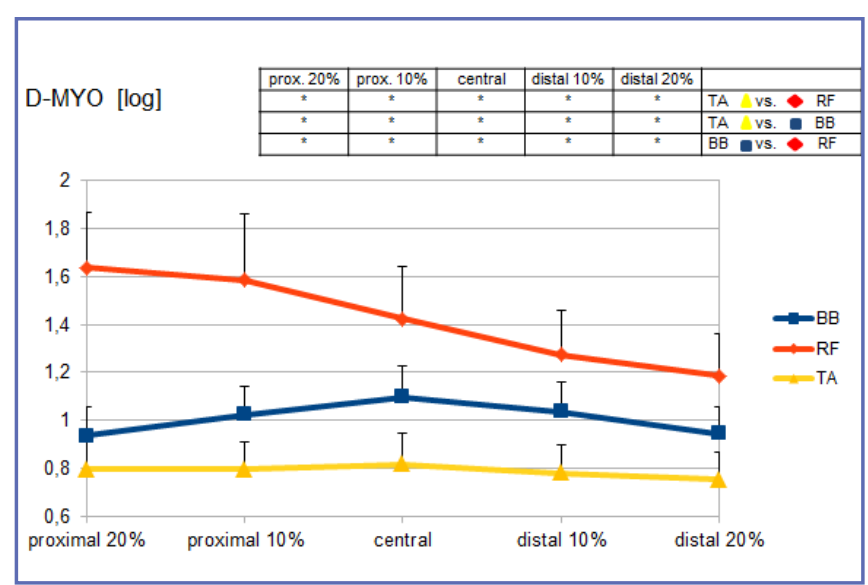

Figure 3. Mean values \pm SD of myometric decrement (D-MYO [log]) at five measurement points (proximal 20\%, proximal $10 \%$, central point, distal $10 \%$ and distal $20 \%$ ) for biceps brachii $(B B$, blue), rectus femoris (RF, red) and tibialis anterior (TA, yellow) muscles and results of pair comparison between muscles (in the upper right corner).

*Statistically significant difference $(\mathrm{P}<0.02)$; NS: not significant. Notice that $Y$ axis does not start at 0 to improve resolution when illustrating differences between muscles.

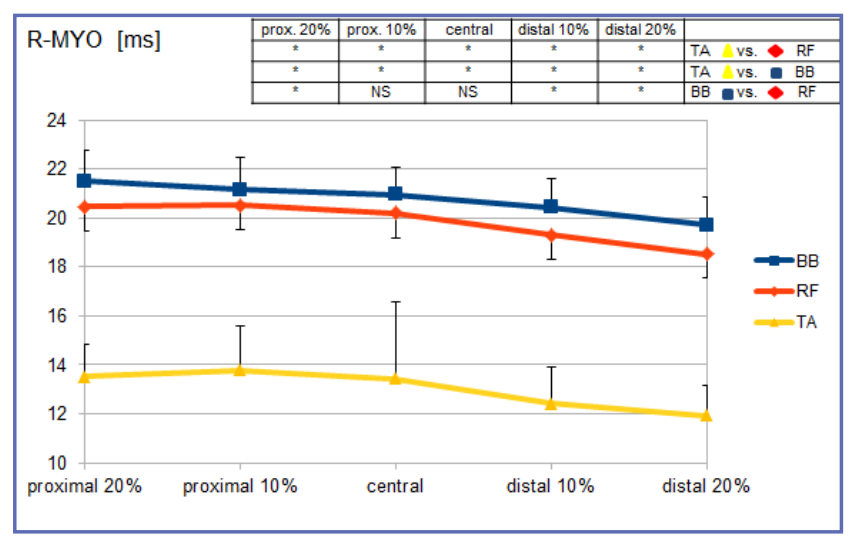

Figure 4. Mean values $\pm \mathrm{SD}$ of myometric relaxation (R-MYO [ms]) at five measurement points (proximal 20\%, proximal $10 \%$, central point, distal $10 \%$ and distal $20 \%$ ) for biceps brachii (BB, blue), rectus femoris (RF, red) and tibialis anterior (TA, yellow) muscles and results of pair comparison between muscles (in the upper right corner).

*Statistically significant difference $(\mathrm{P}<0.02)$; NS: not significant. Notice that $\mathrm{Y}$ axis does not start at 0 to improve resolution when illustrating differences between muscles.

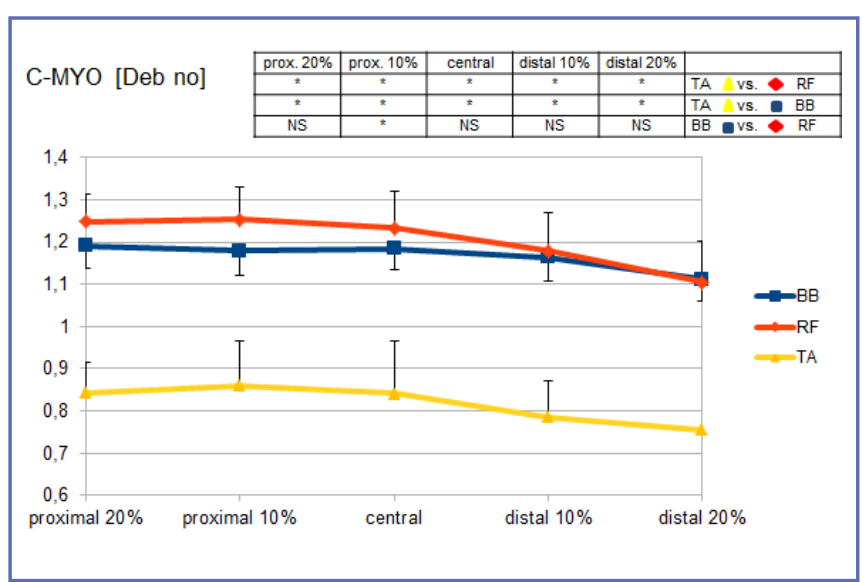

Figure 5. Mean values \pm SD of myometric creep (C-MYO [Deb no]) at five measurement points (proximal 20\%, proximal $10 \%$, central point, distal $10 \%$ and distal $20 \%$ ) for biceps brachii (BB, blue), rectus femoris (RF, red) and tibialis anterior (TA, yellow) muscles and results of pair comparison between muscles (in the upper right corner).

*Statistically significant difference $(\mathrm{P}<0.02)$; NS: not significant. Notice that $\mathrm{Y}$ axis does not start at 0 to improve resolution when illustrating differences between muscles.

\section{DISCUSSION}

\section{Statement of principal findings}

To the best of our knowledge this is the first study showing changes in mechanical properties expressed by five parameters due to changing the position of the myoton probe along the long axes of three skeletal muscles. It is of note that when the myoton probe was moved proximally from the central point the decrement was the only myometric parameter that differentiated the three muscles in their pattern of changes while other parameters did not change significantly irrespective of the muscle tested. When the myoton probe was moved distally from the central point, the relaxation and creep decreased in all three muscles while changes in frequency, stiffness and decrement were muscle dependent. In the $\mathrm{BB}$, central-to-distal relocation of the probe caused an increase in muscle tone and elasticity with no change in stiffness; in the TA there was no change in muscle elasticity and increases in both muscle tone and stiffness, while in the RF muscle no changes in muscle tone or stiffness were observed but there was an increase in muscle elasticity. With the exception of decrement, we observed a generally similar trend of measured parameters along the muscle long axes in the three muscles tested, but there were differences between muscles and measurement points in the parameters of mechanical properties recorded by the Myoton $\mathrm{PRO}^{\circledR}$. The TA muscle has the largest intrinsic tension at 
the cellular level (highest frequency), which partially resulted from its shortest relaxation and smallest creep. The TA was also the most stiff (least compliant) with the greatest elasticity (smallest decrement value) while the RF was the least elastic among the tested muscles. There were much smaller or no differences in some mechanical properties between the RF and the $\mathrm{BB}$ muscles with the exception of muscle elasticity (D-MYO).

\section{Strengths and weaknesses of the study}

To our knowledge, it has not yet been verified whether the change of the measurement point affects the myometric outcomes, including frequency, stiffness, decrement and rarely documented relaxation and creep. Our results show that the outcome measurements differ with the changing position of the myometric probe along the muscle, demonstrating that selection of the measurement point when testing a skeletal muscle affects the values of the myometric parameters. Our results emphasise the need for a standardised measurement point location when using myometry, to enable data obtained to be comparable between studies and indeed, between individuals. The relaxed state of the muscles was not checked and measured by electromyography; therefore, it was not possible to ensure that the limbs were in a resting state, which is a weak point of the study. Other limitations that might influence the results such as age, subcutaneous soft tissue and degree of physical activity on the day of data collection should not affect the measurements (on the day of measurement the subject did not exercise). On the one hand, the subjects were young males, therefore it is unlikely that the subcutaneous soft tissue might influence the results, because the subcutaneous tissue was thin. On the other hand, our study group was relatively homogeneous, therefore observed results should not be generalized to the population.

\section{Strengths and weaknesses in relation to other studies results}

\section{Comparison between measurement points}

In general, in all parameters: muscle tone, stiffness, relaxation and creep did not change significantly when the probe of the myometry device was relocated proximally from the central point, while elasticity (D-MYO) changes were muscle-dependent and varied in $\mathrm{BB}, \mathrm{TA}$ and $\mathrm{RF}$. When the myometry probe was repositioned from a central to a distal $10 \%$ position and especially to a distal $20 \%$ point, the changes in the parameters were more pronounced (except for S-MYO in BB, D-MYO and C-MYO in TA, and F-MYO and S-MYO in RF where the trends in differences were not statistically significant), and in general frequency and stiffness showed increasing values while decrement, relaxation and creep showed decreasing values. The opposite trends observed in changes in myometric parameters at proximal compared to distal points (in respect to the central point) can be at least partially explained in the light of the anatomy of proximal and distal parts of the muscles and differences in elastic properties of tendon and muscle fibres. In some muscles, such the TA and RF, the muscle fibres are attached almost directly to the respective bones at the proximal part with no long tendon (as is the case in the BB). In contrast, at the distal end of the three tested muscles there are longer tendons that are characterized by high stiffness and elasticity (22) and the properties can also vary among muscles due to their function (23). Shadwick showed that differences in material properties between mature flexor and extensor tendons are correlated with their physiological function; the flexor is much better suited to act as an effective biological spring than the extensor (23). The three muscles in our study are mainly flexors; however the RF is a two-way acting muscle as it crosses over the hip and knee joints, and therefore acts as a hip flexor but knee extensor. This is why we noted bigger changes in measured parameters at distal compared to proximal locations.

In the available literature we found only one study by Agyapong-Badu et al. (7), which with some limitations can be compared to our data from the BB and RF for superior and inferior points (because they measured mechanical properties at both medial and lateral points). Depending on the muscle tested $(\mathrm{BB}, \mathrm{RF})$ and measured parameters, their results are in partial agreement with our findings, as discussed in detail below in the section "Comparison between measurement points and muscles". Nevertheless, despite some discrepancies between our data and the results reported by Agyapong-Badu et al. (7), it can be seen that there are opposite changes in myometric parameters when the probe is moved distally or proximally from the central point.

\section{Comparison between muscles}

Despite similarities among muscles in the trends of changes of the measured parameters due to probe relocation along the long axis, the three muscles differed in values of the measured parameters. It can be clearly seen that properties of TA contrast with those of the BB and RF. Generally, the TA was characterized by the greatest muscle tone, stiffness and elasticity, the BB by the lowest tone and stiffness, while the RF was the least elastic. It is of note that despite statistically significant differences between the BB and RF muscles at some measurement points, the values of creep 
and relaxation were close in both muscles. These results indicated that the $\mathrm{BB}$ and RF muscles were characterized by comparable muscle tone, stiffness as well as relaxation and creep. Our findings that the stiffness of the BB was lower than that of the TA or RF agreed with those reported by others $(1,5)$. The values for tone, stiffness, and elasticity (decrement) reported in the present study for the RF and TA muscles were in a range comparable with those found by Fröhlich-Zwahlen et al. (24) in a group of males and females pooled together (age $53 \pm 10$ years). Similar to our findings, the values of stiffness and frequency reported by the authors were higher in the TA than in the RF, while the decrement was smaller in the TA than in the RF. Our results for creep and relaxation in the three muscles cannot be compared with findings of other studies, because to the best of our knowledge, such data have not yet been reported.

The reasons for the high frequency and transverse stiffness and small decrement, creep, and relaxation for the TA are complex but could be related to the muscle's location and smaller anatomical cross-sectional area (CSA) compared with the RF, meaning that bone structure could limit its oscillations, as well as the pinnate muscle architecture and high proportion of type I fibres (25) that are stiffer than those in type II as they are more collagenous (26). It is also suggested that that differences in muscle functions may also be associated with differences in the collagen structure of these muscles. It is supposed that higher compliance (inverse of stiffness) of fast muscle could be partly related to the features and amount of its collagen.

\section{Comparison between measurement points and muscles}

We found in all parameters a significant effect of measurement point and muscle and a significant interaction between measurement point and muscle with the exception of the interaction mentioned in relaxation. We found that in the $\mathrm{BB}$ muscle the elasticity increased when the myoton probe was located at either proximal or distal points, while in the $\mathrm{RF}$ it increased at distal points but decreased at the proximal part of the muscle, and in the TA there were no changes in muscle elasticity. These differences in the changes of the elastic properties (D-MYO) of the three muscles due to probe relocation (especially proximal $20 \%$ and distal $20 \%$ ) may be explained at least partly by the anatomy, architecture and function of the muscles, as well as differences in the elastic properties of tendon and muscle fibres. It has been documented that tendon is characterized by high elasticity (22) and its properties can vary with the function of a muscle (23). The RF and TA muscles have no distinct proximal tendons and at their origin muscle fibres extend from the bone, while the proximal parts of the muscles are thicker and more fleshy than the distal parts, with the CSA being larger in the proximal compared to the distal areas which in the RF muscle is almost twice that of the TA. On the other hand the CSA of distal parts of these muscles is much smaller and the distal tendons are long. The BB muscle has proximal and distal tendons of approximately similar length (circa 8-9 cm and $7.6 \mathrm{~cm}$, respectively) (27). Thus the distal ends of the three muscles are quite similar, having long distal tendons, while the proximal parts differ in both proximal tendon length and CSA. This is why in the BB muscle elasticity increased (D-MYO decreased) at proximal 20\% and distal $20 \%$ (closer to tendons), while at proximal points in the RF elasticity decreased (D-MYO increased) and in the TA did not change. The differences in the elasticity of the RF and TA despite the same muscle fibre architecture (both are pinnate) and similar proximal muscle part can to some extent be explained in the light of differences in CSA. In agreement with our findings Agyapong-Badu and co-authors (7) showed no difference between midpoint and superior (proximal) and inferior (distal) points in the RF for F-MYO and S-MYO while changes in D-MYO (elasticity) were different from ours. We recorded a significant D-MYO increase (elasticity decrease) in RF at proximal points in comparison to the central point. Concerning the BB muscle, our results showing a lack of changes in F-MYO at proximal points and an increase at distal points (compared to the central point) agreed with the study of Agyapong-Badu at el. (7). However, our findings of lack of stiffness (S-MYO) changes at proximal and distal points compared to the central point contrasted to the findings of Agyapong-Badu et al. (7) in matters of distal-to-central point comparison, as they noticed a significant increase in BB stiffness. It is only puzzling that these authors found that the absolute difference in stiffness in the BB muscle between the midpoint and the inferior (distal) part were smaller than in our study $(5$ $\mathrm{N} / \mathrm{m}$ vs $13 \mathrm{~N} / \mathrm{m}$, respectively) but in our study the difference did not reach the level of statistical significance despite similar average and SD values in both studies $(207 \pm 23.22$ $\mathrm{N} / \mathrm{m}$ and $220 \pm 22.24 \mathrm{~N} / \mathrm{m}$ respectively in mentioned study and our study), and the larger number of cases in our study $(\mathrm{n}=32)$ than in their study $(\mathrm{n}=26)$.

Furthermore, the elasticity results (D-MYO) of the BB in our study only partly agreed with those reported by Agyapong-Badu et al. (7) because, compared to the central point, we found a significant decrease in D-MYO at the proximal $20 \%$ and distal $10 \%$ and $20 \%$ points, while they recorded only a decrease at the distal (inferior) point. The discrepancies indicated above between our study and theirs could result to some extent from differences in the distance between the measurement points used in the two studies. Authors mentioned above applied an absolute $1 \mathrm{~cm}$ distance between measurement points $(1 \mathrm{~cm}$ above and $1 \mathrm{~cm}$ below 
the midpoint) on the dominant body side while we marked those points at $10 \%$ of the muscle length and we pooled data of each muscle from the right and left limb of the 16 test subjects that gave the $\mathrm{N}$ equal to 32 .

Nevertheless, despite those discrepancies between our study and that mentioned above, it can be seen that there were opposite changes in myometric parameters when the probe was moved either distally or proximally from the central point.

\section{Meaning of the study: implications for clinicians or policymakers}

Our results showed that selection of measurement points had an impact on the values of the myometric parameters. Differences were observed both for the measurement points located the furthest from each other, as well as for those located adjacent to each other. It means that the results of myometric tests of the same muscles, but measured in different points, should not be compared with each other which is important in the context of research and clinical practice. From a practical point of view, our study emphasizes that the monitoring of the mechanical properties of the muscle over time using myoton device (for example in individuals after a stroke) should be based on the same measuring point. It is also possible to objectively evaluate the effect of the therapy (physiotherapy) or pharmacotherapy on the mechanical properties of muscles, as it was indicated by many studies before $(4,6)$. Our study highlights the importance of standardizing the measuring point (the same before and after the therapy) for this purpose.

The results of our study also showed the variability in muscles' mechanical properties along their long axis, as measured by MyotonPRO ${ }^{\circledR}$ that practitioners should be aware of. The results showed that the muscle properties change more toward distal than proximal end, compared

\section{REFERENCES}

1. Gavronski G, Veraksits A, Vasar E, Maaroos J. Evaluation of viscoelastic parameters of the skeletal muscles in junior triathletes. Physiol Meas 2007;28(6):625-37.

2. Gapeyeva H, Vain A. Methodical Guide: Principles of Applying Myoton in Physical Medicine and Rehabilitation. Muomeetria, Tartu, Estonia, 2008.

3. Gervasi M, Sisti D, Amatori S, et al. Muscular viscoelastic characteristics of athletes participating in the European Master Indoor Athletics Championship. Eur J Appl Physiol 2017;117(8):1739-46. to the central muscle point. Percentage changes in myometric outcomes, for the most distal point, ranged from $5 \%$ to $16 \%$ (except F-MYO and S-MYO in RF muscle), while for the most proximal point, they were less than 6\% (except $\mathrm{D}-\mathrm{MYO}$ in $\mathrm{BB}$ and RF muscle), compared to the central point. It could mean that different methods of muscle therapy (manual therapy, massage, trigger point therapy or fascia therapy) could have different effects on different parts of the muscle (a potentially similar in the proximal and central parts and more variable in the distal part). The knowledge about mentioned variability in mechanical properties, along muscle long axis, might be important for precise botulinum injection navigation, what can be of a great value for therapeutic efficiency.

\section{CONCLUSIONS}

Our results emphasised the variation in muscle properties along the long axis of chosen skeletal muscles expressed by myometry outcomes.

\section{Unanswered questions and future research}

After the significant impact of the location of myometric measurement points on skeletal muscle mechanical properties outcomes has been demonstrated, further research into the real causes are necessary. Future research should focus on establishing the relationship between the differences in measurements observed along the length of a muscle with its objectively assessed structural characteristics. Such determining the causes in directly way, will help to further understand the observed phenomenon.

\section{CONFLICT OF INTERESTS}

The authors declare that thay have no conflict of interests.

4. Lo WLA, Zhao JL, Li L, Mao YR, Huang DF. Relative and Absolute Interrater Reliabilities of a Hand-Held Myotonometer to Quantify Mechanical Muscle Properties in Patients with Acute Stroke in an Inpatient Ward. Biomed Res Int 2017;2017:4294028.

5. Agyapong-Badu S, Warner M, Samuel D, Stokes M. Measurement of ageing effects on muscle tone and mechanical properties of rectus femoris and biceps brachii in healthy males and females using a novel hand-held myometric device. Arch Gerontol Geriatr 2016;62:59-67. 
6. Marusiak J, Jaskólska A, Budrewicz S, et al. Influence of dopaminergic treatment on resting elbow joint angle control mechanisms in patients with Parkinson's disease - a preliminary report. Acta Bioeng Biomech 2018;20(4):75-82.

7. Agyapong-Badu S, Warner M, Samuel D, Stokes M. Practical considerations for standardized recording of muscle mechanical properties using a myometric device: recording site, muscle length, state of contraction and prior activity. J Musculoskel Res 2018;21(2):1850010.

8. Mooney K, Warner M, Stokes M. Symmetry and within-session reliability of mechanical properties of biceps brachii muscles in healthy young adult males using the MyotonPRO device. WPHS 2013;1:1-11.

9. Agyapong-Badu S, Aird L, Bailey L, et al. Interrater reliability of muscle tone, stiffness and elasticity measurements of rectus femoris and biceps brachii in healthy young and older males. WPHS 2013;1(4):1-11.

10. Aird L, Samuel D, Stokes M. Quadriceps muscle tone, elasticity and stiffness in older males: reliability and symmetry using the MyotonPRO. Arch Gerontol Geriatr 2012;55(2):e31-9.

11. Schoenrock B, Zander V, Dern S, et al. Bed Rest, Exercise Countermeasure and Reconditioning Effects on the Human Resting Muscle Tone System. Front Physiol 2018;9:810.

12. Zinder SM, Padua DA. Reliability, validity, and precision of a handheld myometer for assessing in vivo muscle stiffness. J Sport Rehabil 2011;20(3):2010_0051.

13. Mullix J, Warner M, Stokes M. Testing muscle tone and mechanical properties of rectus femoris and biceps femoris using a novel hand held MyotonPRO device: relative ratios and reliability. Work Pap Health Sci 2013;1:1-8.

14. Alnaqeeb MA, Al Zaid NS, Goldspink G. Connective tissue changes and physical properties of developing and ageing skeletal muscle. J Anat 1984;139(Pt 4)(Pt 4):677-89.

15. Ducomps C, Mauriège P, Darche B, Combes $S$, Lebas F, Doutreloux JP. Effects of jump training on passive mechanical stress and stiffness in rabbit skeletal muscle: role of collagen. Acta Physiol Scand 2003;178(3):215-24.

16. Huang J, Qin K, Tang C, et al. Assessment of Passive Stiffness of Medial and Lateral Heads of Gastrocnemius Muscle, Achilles Tendon, and Plantar Fascia at Different Ankle and
Knee Positions Using the MyotonPRO. Med Sci Monit 2018;24:7570-6.

17. Carroll CC. Analgesic Drugs Alter Connective Tissue Remodeling and Mechanical Properties. Exerc Sport Sci Rev 2016;44(1):29-36.

18. Hoang PD, Herbert RD, Todd G, Gorman RB, Gandevia SC. Passive mechanical properties of human gastrocnemius muscle tendon units, muscle fascicles and tendons in vivo. J Exp Biol 2007;210(Pt 23):4159-68.

19. Oldfield RC. The assessment and analysis of handedness: The Edinburgh Inventory. Neuropsychologia 1971;9:97-113.

20. van Melick N, Meddeler BM, Hoogeboom TJ, Nijhuis-van der Sanden MWG, van Cingel REH. Nijhuis-van der Sanden, M. W. G., van Cingel, R. E. H. How to determine leg dominance: The agreement between self-reported and observed performance in healthy adults. PLoS One 2017;29;12(12):e0189876.

21. Padulo J, Oliva F, Frizziero A, Maffulli N. Muscles, Ligaments and Tendons Journal - Basic principles and recommendations in clinical and field Science Research: 2018 update. Muscles Ligaments Tendons J 2018;8:305-7.

22. Alexander RM. Tendon elasticity and muscle function. Comparative Biochemistry And Physiology A-Molecular \& Integrative Physiology 2002;133(4):1001-11.

23. Shadwick RE. Elastic energy storage in tendons: Mechanical differences related to function and age. J App Physiol 1990;68:1033-40.

24. Fröhlich-Zwahlen AK, Casartelli NC, Item-Glatthorn JF, Maffiuletti NA. Validity of resting myotonometric assessment of lower extremity muscles in chronic stroke patients with limited hypertonia: a preliminary study. J Electromyogr Kinesiol 2014;24:762-9.

25. Johnson MA, Polgar J, Weightman D, Appleton D. Data on the distribution of fibre types in thirty-six human muscles. An autopsy study. J Neurol Sci 1973;18(1):111-29.

26. Kovanen V, Suominen H, Heikkinen E. Collagen of slow twitch and fast twitch muscle fibers in different types of rat skeletal muscle. Eur J Appl Physiol Occup Physiol 1984;52:235-42.

27. Joshi S, Joshi YA, Sontakke PS, Mittal A. Some details of morphology of biceps brachii and its functional relevance. J Anat Soc India 2014;63(1):24-9. 